\title{
Verleihung des Bracco-Poster-Preises 2018
}

Auf dem 99. Deutschen Röntgenkongress in Leipzig wurde erneut der Bracco-PosterPreis verliehen. Ausgezeichnet wurde Herr Dr. Markus Lerchbaumer vom Institut für Radiologie der Charité-Universitätsmedizin in Berlin für den Beitrag „Stellenwert der kontrastmittelunterstützten Sonografie in der Evaluation von Nierenzysten unter Anwendung der Bosniak-Klassifikation im Vergleich zur Computer- und Magnetresonanztomografie“.

Der Jury gehörten Prof. Dr. Peter Huppert, Prof. Dr. Peter Landwehr und Prof. Dr. Stefan Delorme an.

Der Bracco-Poster-Preis wird für hervorragende Poster auf dem zukunftsträchtigen und innovativen Gebiet des kontrastverstärkten Ultraschalls vergeben. Er ist mit 1000 Euro dotiert und wird von der Firma Bracco Imaging gestiftet. 